\title{
Innovation Agile : Regards Pluridisciplinaires
}

\section{Agile Innovation: Multidisciplinary Approaches}

\author{
Laurent Dupont $^{1}$, Laure Morel $^{1}$ \\ ${ }^{1}$ Université de Lorraine, Laboratoire ERPI, France, I.dupont@univ-lorraine.fr, laure.morel@univ-lorraine.fr
}

RÉSUMÉ. Un collectif de scientifique a posé son regard pluridisciplinaire sur l'innovation agile. Ce concept, parfois perçu comme un effet de mode, n'avait jusqu'à présent jamais fait l'objet d'une mise en débat extensive et aussi diversifiée par une large communauté scientifique. Les premières observations montrent que l'innovation agile reflète les questionnements des organisations petites ou grandes, publiques ou privées, individuelles ou regroupées face aux évolutions qu'elles ont dû mettre en place pour répondre aux défis sociétaux, économiques ou environnementaux actuels. II fait donc sens de travailler et de s'interroger collectivement sur l'innovation agile et son impact sur les processus et les dynamiques d'innovation. Les six articles présentent un regard croisé issu de plusieurs disciplines telles que l'ingénierie de l'innovation, les sciences de gestion et l'économie. Chacun explore un domaine différent ou une piste circonscrite pour apporter sa pierre à la compréhension de ce concept complexe. Mis en commun, ces articles créent une mosaïque subtile proposant autant d'éclairages originaux et complémentaires pour esquisser un panorama des connaissances théoriques et pratiques en matière d'innovation agile et d'agilité.

ABSTRACT. A group of scientists have decided to take a multidisciplinary look at agile innovation. This concept, sometimes perceived as fashionable, has never before been the subject of such an extensive and diversified debate in the scientific community. Initial observations show that agile innovation reflects the questioning of companies/organizations both small and large, public and private, individual and grouped, due to the changes they need to implement in order to respond to current societal, economic and environmental challenges.

It therefore makes sense to collectively work on and question agile innovation and its impact on processes and innovative dynamics. The six articles presented combine several research fields: innovation engineering, management sciences and economics. Each explores a different aspect or a specific path to help us understand this complex concept. Together, these articles create a subtle mosaic of original and complementary insights to sketch a panorama of theoretical and practical knowledge in agile innovation and agility.

MOTS-CLÉS. Innovation agile, Pluridisciplinarité, Agilité, Organisation, Ingénierie, Gestion, Économie.

KEYWORDS. Agile Innovation, Multidisciplinary, Agility, Organization, Engineering, Management, Economy.

Aux grands défis énergétiques, écologiques, numériques, économiques et sociétaux, les organisations - entreprises, institutions, territoires - répondent par l'innovation pour créer de la valeur. Elles développent notamment leur capacité à innover [GAL 15], en combinant des activités d'innovation incrémentale à des projets d'innovation de plus en plus performants, voire de rupture. Ceci se traduit aujourd'hui par l'intégration du concept «d'innovation agile » aux grandes problématique de société [AAE 08, MAR 10, MER 16, PIR 10, WIL 11]. Ce numéro spécial de TechInn rend compte de réflexions issues de plusieurs domaines de recherche : l'ingénierie de l'innovation, les sciences de gestion et à l'économie. Ce dernier présente un aperçu de la richesse des domaines d'expertise couverts par la communauté du Réseau de Recherche sur l'Innovation, dont la mobilisation permet d'éclairer subtilement et de façon complémentaire les études et réflexions relatives à l'innovation ou aux méthodes agiles. Le lecteur doit dès lors faire preuve d'agilité intellectuelle pour plonger dans la diversité des paradigmes mobilisés.

Au premier abord, l'innovation agile paraît être soit un pléonasme, soit une nouvelle qualification de l'innovation. Néanmoins, loin d'être réducteur, le terme «agile » révèle certains des enjeux actuels pour les acteurs intéressés par l'innovation. Une définition proposée par le CNRLT esquisse certain des défis associés. Au sens figuré ce terme signifie "vif, prompt à comprendre ou à saisir », en parlant d'un mouvement de l'intelligence ${ }^{1}$. L'accélération que semble vivre notre société requière à la fois de la

\footnotetext{
${ }^{1}$ Consulté le 20 août 2018
} 
rapidité, de la réactivité et une capacité à comprendre la dimension systémique des défis et des projets à mettre en ouvre quel que soit le secteur ou le type d'organisations et d'acteurs concernés. Nous cherchons dès lors à qualifier et comprendre les contours de ce nouveau contexte. Cette nécessaire maturité en matière d'analyse peut se définir comme la capacité d'une organisation à s'immerger de manière effective dans son écosystème, entendu comme l'ensemble des entités qui interagissent dans un environnement technologique, économique et sociétal. Les structures concernées associent dès lors systématiquement toutes parties prenantes à la réalisation de nouveaux produits et/ou services, afin d'accélérer la compréhension de leurs différents besoins et de ce fait, de développer une innovation et une création de valeur ajoutée y répondant spécifiquement.

Pour atteindre ce degré d'innovation, les organisations doivent adapter leurs stratégies, leurs modèles d'affaires, leurs projets, voire redéfinir leur rôle au sein de leur " écosystème ». Ainsi, les entreprises sont, par exemple, dans l'obligation à la fois d'engager des projets de transformation de leurs organisations, de leurs méthodes de travail et de leurs moyens de production en s'ouvrant à leur environnement tout en continuant à réaliser des projets innovants leur permettant de rester compétitifs en développant des nouveaux produits et/ou services. Les territoires quant à eux redessinent leurs compétences et leurs relations avec leurs partenaires, tout en imaginant de nouvelles ressources pour concevoir et faire vivre leurs projets locaux, interdépendants de projets nationaux, voire internationaux.

Ces situations génèrent un fort degré d'incertitude tant dans leur mise en œuvre que dans leurs perspectives d'aboutissement. La transformation de notre société implique donc de gérer les nouveaux défis, voire des ruptures, grâce d'une part au développement d'innovations et d'autre part via la mise en place d'organisations flexibles et adaptatives, c'est à dire agiles. Le concept "d'agilité » se développe et est popularisé depuis le début des années 2000 à travers les méthodes agiles et son manifeste conçu pour le développement de logiciels [AGI 01, HIG 01]. Ce dernier met en avant trois principes fondamentaux : (1) Les interactions entre les individus supportées par des processus et des outils agiles. En effet, dans l'optique agile, l'équipe est bien plus importante que les outils (structurants ou de contrôle) ou les procédures de fonctionnement. Il est préférable d'avoir une équipe soudée et qui communique plutôt qu'une équipe composée d'experts fonctionnant chacun de manière isolée. (2) Une collaboration avec les clients qui prend la forme d'une contractualisation souple. Le client doit être impliqué tout au long du développement. Il ne fait plus sens de négocier et figer un contrat au début du projet, alors que les demandes du client peuvent évoluer au gré de la compréhension de ses besoins et du champ des possibles. Dans ce cadre, la notion même de client peut sans doute être repensée. Les clients, comme l'ensemble des parties prenantes d'un projet, en constituent la richesse. Les inclure dans le processus est un des piliers d'une « robuste agilité ». (3) L'adaptation au changement, voire l'incarnation du changement, plus que le suivi d'un plan. Dans un contexte accéléré, où l'écosystème peut inhiber des pistes ou offrir de nouvelles opportunités dans des temps courts, a minima, la planification initiale et la structure du produit ou service doivent être flexibles afin de s'adapter à l'évolution de la demande du client (ou du marché). De façon plus ambitieuse, il est nécessaire d'acquérir et diffuser une logique de proactivité, pour concevoir ensemble le futur souhaité.

Dès lors, il semble qu'inscrire des organisations dans une dynamique d'agilité, c'est leur permettre de créer de la valeur en adoptant une culture du changement et une logique de collaborations aussi bien en interne qu'à l'externe. L'agilité transforme les organisations sur trois niveaux : (1) la réalisation de projets innovants qui bénéficient des méthodes agiles puisque ces dernières permettent de diminuer les craintes et les incertitudes en les intégrant directement aux processus de conception et de développement et de les envisager comme opportunités de progrès ; (2) la mise en place d'un environnement créatif et accélérateur d'innovation qui permette de transformer une simple idée en un produit / service approprié par les parties-prenantes ; (3) la gestion et l'évaluation plus efficace du processus même d'innovation pouvant transformer le modèle organisationnel, voire la filière dans laquelle s'intègre l'organisation adoptant l'agilité. 
Sans être exhaustif, les six articles de ce numéro spécial illustrent et détaillent de manière complémentaire des questionnements sous-jacents à l'émergence de l'innovation agile, ainsi que des pistes de développement possibles face aux différentes problématiques identifiées à différentes échelles et pour différents contextes.

Stéphane Goria explore la relation créativité-veille et suggère d'employer une boucle OODA (Observation, Orientation, Décision, Action) pour en estimer l'agilité.

Laurent Dupont et ses co-auteurs rappellent le rôle fondamental de la confiance au sein des processus d'innovation. Via deux études de cas dans le domaine de l'énergie, ils décrivent en quoi une nouvelle technologie innovante peut soutenir ou freiner la confiance et donc l'agilité au sein d'un processus de co-création.

Sur la base du cas illustre de la montre Swatch, Brunelle Marche et ses collègues montrent l'existence d'un lien fort en innovation et filière et font émerger les notions de filière agile et d'agilité de filière au service de l'innovation.

Les travaux d'Hélène Perrin-Boulonne appréhendent le duo «entrepreneur investisseur » comme la conception agile d'une relation au sein d'un système territorial d'innovation.

Manon Enjolras et ses co-auteurs rappellent le caractère dynamique et évolutif de la notion d'innovation, impliquant de développer une méthodologie de mise à jour itérative des indicateurs de mesure de la capacité à innover. L'évaluation de l'innovation doit être agile.

Enfin, Michel Sonntag questionne le caractère pérenne ou éphémère du concept d'agilité. Il souligne l'existence d'attentes plus ou moins exprimées derrière cette notion, notamment en termes de mobilisation des compétences individuelles et collectives présentes ou nécessaires à la survenue d'une l'innovation.

Ces premiers travaux sont une invitation à poursuivre les réflexions pour renforcer la connaissance et la compréhension de l'innovation agile. De plus, ils se veulent une réponse opérationnelle à destination des praticiens, des entreprises et des territoires qui souhaitent favoriser l'innovation agile ou l'agilité de l'innovation comme une invitation à explorer avec souplesse et intelligence d'autres horizons...

\section{Bibliographie}

[AAE, 08] AAEN, I., Essence: Facilitating agile innovation, In International Conference on Agile Processes and Extreme Programming in Software Engineering, 1-10, Springer, 2008.

[Agi 01] AgILE AlLianCE, « Manifesto for Agile Software Development », 2001.

[GAL15] GALVEZ MANRIQUEZ, D., Évaluation de la capacité à innover : une approche par auto évaluation et suivi supporté par des analyses multicritères dynamiques, Université de Lorraine, Nancy, 2015.

[Hig 01] HighSmith, J., Fowler, M., « The Agile Manifesto », Software Development Magazine, 9(8), p. 29-30, 2001.

[Mar 10] Margaria, T., STEFfen, B., « Simplicity as a Driver for Agile Innovation », Computer, 43(6), p. 90-92, 2010.

[Mer 16] MERGEL, I., " Agile Innovation Management in Government: A Research Agenda », Government Information Quarterly, 33(3), p. 516-523, 2016.

[PIR 10] PIROla-Merlo, A., « Agile Innovation: The Role of Team Climate in Rapid Research and Development », Journal of Occupational and Organizational Psychology, 83(4), p. 1075-1084, 2010.

[WIL 11] WILSON, K., DoZ, Y. L., « Agile Innovation: A Footprint Balancing Distance and Immersion », California Management Review, 53(2), p. 6-26, 2011. 